\title{
Posturografia em idosos com distúrbios vestibulares e quedas
}

\section{Posturography in elderly people with vestibular disorder and falls}

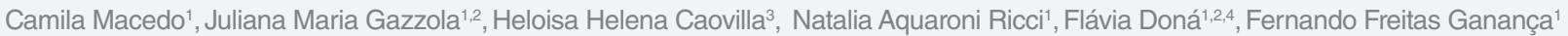 \\ 'Departamento de Otorrinolaringologia e Cirurgia de Cabeça e Pescoço da UNIFESP - São Paulo (SP), Brasil. \\ 2Programa de Mestrado Profissional em Reabilitação do Equilíbrio Corporal e Inclusão Social da Universidade Bandeirante de São Paulo (UNIBAN) - \\ São Paulo (SP), Brasil. \\ ${ }^{3}$ Departamento de Fonoaudiologia da UNIFESP - São Paulo (SP), Brasil. \\ ${ }^{4}$ Departamento de Neurologia/Neurocirurgia da UNIFESP - São Paulo (SP), Brasil.
}

DOI: http://dx.doi.org/10.7322/abcshs.v38i1.4

\section{RESUMO}

Introdução: A posturografia estática e dinâmica tem sido usada para analisar a habilidade de idosos com disfunção vestibular em manter o equilíbrio corporal em diferentes condições de conflitos sensoriais. O objetivo do exame é quantificar a velocidade de oscilação e o deslocamento do centro de pressão nas condições de conflitos visual, somatossensorial e interação visuo-vestibular, e o limite de estabilidade. Objetivo: Analisar a literatura referente ao controle do equilíbrio corporal em idosos com distúrbios vestibulares por meio de posturografia computadorizada estática e dinâmica. Métodos: Revisão nas bases de dados LILACS, EMBASE, MEDLINE, Scielo, Cochrane, ISI Web of Knowledge e bibliotecas virtuais de teses e dissertações, utilizando as palavras-chave "Idoso", "Equilíbrio Postural", "Avaliação", "Controle Postural", "Quedas", "Posturografia", "Vestibular" e/ou "Tontura" de publicações dos últimos vinte anos. Resultados: Há vários modelos de posturografias que mensuram as respostas posturais e o risco de quedas em indivíduos idosos, perante os diferentes estímulos sensoriais, incluindo a tecnologia de realidade virtual. Os idosos com desequilíbrio corporal, tontura, e/ou com histórico de quedas apresentam pior desempenho que idosos sem queixas, sem histórico de quedas e indivíduos mais jovens. Conclusão: A posturografia é uma valiosa ferramenta para análise quantitativa do controle postural, permitindo a identificação das condições sensoriais nas quais os idosos vestibulopatas apresentam maior instabilidade.

Palavras-chave: idoso; equilíbrio postural; avaliação; vestíbulo do labirinto; acidentes por quedas; tontura.

\begin{abstract}
Introduction: Static and dynamic posturography has been used to investigate the ability of older people with vestibular disorders to maintain balance under varying conflicting sensory conditions. The goal of testing is to quantify the velocity of oscillation and the center of pressure displacement in different conditions of visual, somatosensory and visual-vestibular conflicts, and the limit of stability. Objective: To analyze the literature of the quantitative assessment of postural stability and balance using static and dynamic computerized posturography in elderly patients with vestibular dysfunction. Methods: A research was performed in the databases LILACS, EMBASE, MEDLINE, Scielo, Cochrane, ISI Web of Knowledge and virtual libraries of theses and dissertations in publications of the last 20 years, using the keywords: "elderly", "Postural Balance", "Postural Control", "Assessment", "Falls", "Posturography", "Vestibular" and / or dizziness." Results: There are several models of computerized posturography that measure the postural responses and the fall risk in older individuals, in different environmental sensory stimuli, including virtual reality technology. Elderly people with imbalance, dizziness, and/or a history of falls have worse performance than elderly with no complaints and no history of falls and younger subjects. Conclusion: The posturography is a valuable tool for quantitative analysis of postural control, and it allows identify the sensory conditions in which the elderly with vestibular disorders have major instability.
\end{abstract}

Keywords: aged; postural balance; evaluation; vestibule, labyrinth; accidental falls; dizziness. 


\section{INTRODUÇÃO}

As quedas e o desequilíbrio corporal nos idosos são decorrentes de alterações associadas a várias doenças crônico-degenerativas e diminuição da reserva funcional dos sistemas sensório-motores envolvidos no mecanismo do Controle Postural (CP) - sensorial (visual, somatossensorial e vestibular), efetor (tônus e força muscular, amplitude de movimento, alinhamento biomecânico e flexibilidade) e processamento central ${ }^{1,2}$. Outros fatores que podem alterar o CP são déficit cognitivo, distúrbio do sono, abuso de bebidas alcoólicas, medicamentos psicoativos, medicamentos ototóxicos, entre outros ${ }^{2}$.

$\mathrm{Na}$ avaliação do equilíbrio, o avaliador deve escolher o tipo de abordagem mais adequado à finalidade da investigação: abordagem funcional, por sistemas ou posturográfica ${ }^{3}$. A posturografia, também chamada de estabilometria, estabilografia ou estatocinesiografia, é capaz de mensurar a excursão ântero-posterior (AP) e médio-lateral (ML) do corpo, por meio de sensores que transformam as oscilações mecânicas das forças de atrito dos pés com a plataforma em sinais elétricos que são amplificados, gravados e analisados. Portanto, os sistemas de plataforma de força podem quantificar objetivamente a velocidade de oscilação corporal (VOC) e a área de deslocamento do corpo pela medida do centro de pressão (COP) relacionada com a base de sustentação ${ }^{4}$ e ativação muscular ${ }^{4,5}$.

O COP é o ponto de aplicação das resultantes das forças verticais agindo na superfície de suporte, e representa um resultado coletivo do sistema de CP e da força gravitacional ${ }^{6}$. A amplitude de movimento do COP é inversamente proporcional ao desempenho do controle do equilíbrio, ou seja, quanto maior a área de deslocamento do COP e a VOC, maior o grau de instabilidade postural ${ }^{2}$.

Várias evidências da utilidade clínica da posturografia foram descritas, constituindo um teste para o diagnóstico diferencial dos distúrbios do equilíbrio corporal em diferentes grupos ${ }^{7,8}$, para a avaliação diagnóstica do comprometimento vestibular ${ }^{9,10}$, caracterização de sensibilidade e especificidade do equipamento para o diagnóstico clínico ${ }^{11,12}$, análise de instabilidade de idosos ${ }^{2,13}$, identificação de risco de quedas ${ }^{14-16} \mathrm{e}$ avaliação dos efeitos de intervenções terapêuticas ${ }^{17}$.

O objetivo deste estudo é analisar a literatura referente ao controle do equilíbrio corporal em idosos com distúrbios vestibulares por meio de posturografia computadorizada estática e dinâmica.

\section{MÉTODO}

Foi realiza revisão nas bases de dados LILACS, EMBASE, MEDLINE, Scielo, Cochrane, ISI Web of Knowledge e bibliotecas virtuais de teses e dissertações, utilizando as palavras-chave "Idoso", "Equilíbrio Postural", "Avaliação", "Controle Postural", "Quedas", "Posturografia”, "Vestibular" e/ou “Tontura” de publicações dos últimos 20 anos (1990-2010).

\section{RESULTADO E DISCUSSÃO}

Vários modelos de posturografia estática e dinâmica são disponíveis no mercado, como Equitest System ${ }^{\circledR}$, Biodex Balance System $^{\circledR}$, sistema de posturografia NedSVE/IBV ${ }^{\circledR}$, Smart Balance Master $^{\oplus}$, Chattecx Balance System ${ }^{\circledast}$, AMTI Plataform, Test for Equilibrium Under Altered Sensory Conditions (TEUSAC), Mumedia's Statitest ${ }^{\circledR}$ e Balance Rehabilitation Unit ${ }^{\circledR}\left(\mathrm{BRU}^{\circledR}\right)$, entre outros. Os modelos diferem quanto aos tipos de informações sensoriais, como o uso de plataformas móveis ou da tecnologia de realidade virtual para recriarem ambientes e situações capazes de medir as respostas posturais do indivíduo perante os diferentes estímulos. Estes modelos apresentam diversos tipos de protocolos de análise do controle postural ${ }^{2}$.

Os programas mais utilizados consistem: (1) teste de Controle Motor com o registro de respostas de movimentos da superfície de suporte; e (2) teste de organização sensorial com o registro do equilíbrio corporal em condições sensoriais ${ }^{18}$. Em pacientes vestibulopatas, o teste de organização sensorial (TOS) é o programa de avaliação posturográfica mais importante, já que consegue manipular e analisar a entrada e dependência das informações dos três sistemas sensoriais (visual, somatossensorial, vestibular) para o equilíbrio corporal ${ }^{18}$.

Foram encontrados 12 estudos científicos em que a posturografia computadorizada foi utilizada na avaliação do controle do equilíbrio corporal de idosos com distúrbios vestibulares. O Quadro 1 apresenta de forma resumida os principais resultados desta pesquisa.

Baloh et al. ${ }^{7}$ avaliaram 140 idosos divididos em dois grupos (indivíduos com queixa de desequilíbrio e/ou tontura e indivíduos que consideraram seu equilíbrio normal para a idade) por meio da posturografia estática e dinâmica da Chattecx Balance System ${ }^{\circledR}$ pelo parâmetro da VOC ântero-posterior (AP) e médio-lateral (ML). As condições avaliadas foram: 1 - plataforma estática; 2 - plataforma estática com espuma; 3 - plataforma com tilt na direção AP; e, 4 - plataforma com tilt na direção ML. Todas as condições foram testadas com os olhos abertos (OA) e olhos fechados (OF). Os resultados mostraram um aumento da VOC do grupo de idosos com queixa de desequilíbrio e/ou tontura em comparação ao controle com diferença significante nas condições: $1 \mathrm{OA}$ e OF para VOC AP; 2 OA para VOC AP, 3 OA para VOC AP e ML, 4 OA para VOC AP e ML e OF para VOC AP. O relato de quedas no último ano do grupo de idosos com queixa foi de $60,0 \%$ e do grupo controle de $39,0 \%$.

Em 1998, Baloh et al. ${ }^{8}$ realizaram nova avaliação comparando três grupos: 70 idosos ( $\geq 75$ anos) que consideraram seu equilíbrio normal, 70 idosos com distúrbios vestibulares periféricos/centrais e com tontura e/ou desequilíbrio de causas desconhecidas e 30 jovens normais. Novamente os autores avaliaram a VOC pela posturografia Chattecx Balance System ${ }^{\circledR}$. Em todas as mensurações, o grupo de jovens obteve menor VOC do que os idosos, independentemente da queixa, e esta diferença foi significativamente maior para os testes dinâmicos quando comparados aos testes estáticos. A análise de interação com a variável "gênero" não foi 
Quadro 1: Principais resultados dos artigos pesquisados

\begin{tabular}{|c|c|}
\hline Autor & Principais achados \\
\hline Furman $^{19}$ & $\begin{array}{l}\text { O TOS da Posturografia Dinâmica Computadorizada (PDC) de adultos assintomáticos mostrou um declínio } \\
\text { significante no escore total associado à idade e às mudanças nas estratégias de movimento. O desempenho foi } \\
\text { significativamente melhor nos grupos com menor faixa etária em relação aos grupos mais idosos em todas as } \\
\text { condições sensoriais. }\end{array}$ \\
\hline Baloh et al. ${ }^{7}$ & $\begin{array}{l}\text { Os resultados mostram um aumento da VOC nos idosos com queixa de desequilíbrio e/ou tontura em relação aos } \\
\text { grupos de idosos sem queixas. As diferenças significantes foram encontradas nas condições: estática sem espuma } \\
\text { com olhos abertos (OA) e olhos fechados-OF (AP); estática sobre espuma com OA (AP), plataforma com tilt na } \\
\text { direção AP com OA (AP e ML) e plataforma com tilt na direção ML com OA (AP e ML) e OF (AP), por meio da } \\
\text { avaliação posturográfica da Chattecx Balance System }{ }^{\circledast} \text {. }\end{array}$ \\
\hline Baloh et al. ${ }^{8}$ & $\begin{array}{l}\text { A VOC da posturografia estática e dinâmica da Chattecx Balance System }{ }^{\oplus} \text { mostrou que o grupo de sujeitos jovens } \\
\text { obteve significativamente maiores pontuações. Houve diferença significativamente maior da VOC dos idosos } \\
\text { com distúrbios vestibulares em relação aos com equilíbrio normal nos testes dinâmicos. Em contraste, nos testes } \\
\text { estáticos, esta medida é aproximadamente igual em ambos os grupos. }\end{array}$ \\
\hline Baloh et al. ${ }^{20}$ & $\begin{array}{l}\text { Durante o seguimento de três anos de idosos com equilíbrio inicialmente normal, foi observado aumento significante } \\
\text { nos valores da VOC, embora não tenham sido registrados aumentos significativos nos valores da amplitude } \\
\text { de oscilação. Também não foram observadas diferenças quanto à velocidade, à amplitude de oscilação entre } \\
\text { os indivíduos que caíram ou não no ano anterior ou no ano após a queda relatada e nem quanto às diferentes } \\
\text { condições de teste (OA e OF) e nas direções ML e AP, por meio da Chattecx Balance System }{ }^{\circledR} \text {. }\end{array}$ \\
\hline Wallmann ${ }^{21}$ & $\begin{array}{l}\text { Ao comparar idosos que não sofreram quedas e aos que sofreram, não foi encontrada diferença significante entre } \\
\text { o Functional Reach Test e o limite de estabilidade (LE) da PDC. No entanto, o grupo que sofreu quedas teve uma } \\
\text { média significativamente menor no escore da condição } 4 \text { do TOS. }\end{array}$ \\
\hline Buatois et al. ${ }^{22}$ & $\begin{array}{l}\text { Ao comparar } 3 \text { grupos de idosos (não caiu, caiu uma vez e duas ou mais vezes) todos saudáveis e funcionalmente } \\
\text { ativos, não observou diferenças significativas quanto às variáveis: idade, gênero, auto-avaliação do estado atual } \\
\text { de saúde, atividade física, índice de massa corporal, avaliação cognitiva, medo de quedas e a história prévia de } \\
\text { queda, e nos testes clínicos-funcionais (Timed Up and Go test, One-Leg Balance e Sit-to-Stand-test) e estáticos e } \\
\text { dinâmicos da PDC. No entanto, os caidores recorrentes apresentaram pior desempenho em relação aos idosos que } \\
\text { caíram uma vez e, estes apresentaram melhor CP em relação aos que não caíram tanto no escore de equilíbrio da } \\
\text { condição } 2 \text { (OF e SF) do TOS como na análise somatossensorial, por meio da PDC. As medidas que diferenciaram } \\
\text { melhor o equilíbrio corporal, em conjunto por análise de regressão logística, entre idosos com quedas recorrentes } \\
\text { e que não caíram, foram a terceira tentativa da condição } 6 \text { (plataforma móvel e campo visual móvel) e a análise } \\
\text { somatossensorial. }\end{array}$ \\
\hline
\end{tabular}

Mostrou que a velocidade média ML e a amplitude do deslocamento ML do COP na posição ortostática em SF com $\mathrm{OA}$ ou OF foram preditoras de quedas em idosos quando mensuradas em plataforma de força. As medidas destes parâmetros na posturografia dinâmica não foram consideradas preditivas de quedas em idosos, neste estudo de revisão sistemática.

Ao comparar o CP, por meio da posturografia estática do NedSVE/IBV ${ }^{\circledR}$, de idosos sem distúrbios vestibulares com os que possuíam tais distúrbios, verificou-se melhor desempenho do grupo sem distúrbios em todos os testes. Os pacientes com distúrbios centrais tiveram resultados piores que os idosos com doença periférica e, destes, os pacientes descompensados foram piores. Os valores de oscilação aumentaram, respectivamente, nas condições de Romberg com OA, Romberg com OF, Romberg com OA em espuma e Romberg com OF em espuma. Observou ainda que todos os resultados da posturografia estática (exceto para o parâmetro de velocidade média) correlacionaram significativamente com os resultados da Berg Balance Scale nas condições de Romberg com OA e com OF, e sobre a espuma com OA.

Os idosos com instabilidade e quedas no último ano mostraram valores inferiores do LE e valores superiores da área do COP e da VOC nas diferentes condições avaliadas de estimulação sensorial (SF, OA, campo visual estático: campo visual dinâmico - perseguição ocular lenta, estímulos sacádicos, estímulos optocinéticos e IVV), quando comparados a voluntários saudáveis, na avaliação pela Balance Rehabilitation Unit.

Os resultados posturográficos, avaliados pela PDC, foram piores no grupo de idosos sintomáticos quando

Pedalini et al ${ }^{13} \quad$ comparados ao grupo de idosos sem queixa, revelando que as alterações vestibulares associadas à senescência comprometem o CP. Além disso, os resultados do grupo de idosos sem queixa foram piores em relação ao grupo de adultos saudáveis.

À avaliação da Balance Rehabilitation Unit, em dez condições sensoriais, idosos saudáveis apresentaram LE melhor do que idosos vestibulopatas crônicos com ou sem quedas; idosos saudáveis apresentaram área do COP e VOC similares à de idosos vestibulopatas crônicos sem quedas e melhores em relação àqueles que apresentaram quedas.

Idosos com distúrbio vestibular central e histórico de desequilíbrio e duas ou mais quedas no último ano apresentam maior dependência visual e risco de queda, quando submetidos à estimulação visual (optocinética e foveal) e avaliados por meio da plataforma AMTI Accusway, pois se verificou aumento na área do COP, aumento significativo na $V O C$ e maiores frequências de oscilação, em relação ao grupo controle. 
significativa, reforçando que os efeitos estão relacionados ao envelhecimento. Houve diferença significativamente maior da VOC dos idosos com distúrbios vestibulares em relação aos idosos com equilíbrio normal nos testes dinâmicos. Em contraste, nos testes estáticos esta medida foi aproximadamente igual em ambos os grupos. Os dados da posturografia não se correlacionaram com a frequência de quedas relatadas no último ano em todos os grupos.

Furman ${ }^{19}$ avaliou o protocolo TOS por meio da posturografia de 94 adultos assintomáticos, distribuídos em quatro grupos: adultos jovens (18 a 44 anos), adultos de meia idade ( 45 a 59 anos), idosos (60 a 79 anos) e muito idosos ( 80 a 89 anos). Os resultados mostraram declínio significante no escore total associado à idade $\mathrm{e}$ às mudanças nas estratégias de movimento. Segundo Furman, o desempenho foi significativamente melhor nos grupos com menor faixa etária em relação aos grupos mais idosos em todas as condições sensoriais. Estes dados podem refletir que mudanças relacionadas à idade, tais como alterações anatômicas do sistema vestibular, tornam-se mais evidentes com o aumento da idade, mesmo em indivíduos assintomáticos, não sendo uniformes dentro das faixas etárias ${ }^{19}$.

Ao realizar uma avaliação pela posturografia Chattecx Balance System $^{\circledR}$, por um período de três anos, em outra investigação, Baloh et al. ${ }^{20}$ analisaram a VOC de 72 idosos (idade média de 82,3 anos) que relataram inicialmente equilíbrio normal para a idade e eram funcionalmente independentes. Durante o seguimento, foi observado pelos autores, um aumento significante nos valores da VOC, embora não tenham sido registrados aumentos significativos nos valores da amplitude de oscilação. Os autores também não encontraram diferença significante quanto à velocidade, à amplitude de oscilação entre os indivíduos que caíram ou não no ano anterior ou no ano após a queda relatada e nem quanto às diferentes condições de teste (OA e OF) e nas direções ML e AP. Durante o seguimento, um pequeno subgrupo apresentou alterações nas condições clínicas e nos testes de equilíbrio funcional e aumento na velocidade de oscilação na posturografia ${ }^{20}$.

Em 2001, Wallmann ${ }^{21}$ avaliou 15 idosos da comunidade que não sofreram quedas (média etária 74,9 anos) e 10 idosos que sofreram quedas (média etária de 72,7 anos) por meio do Functional Reach Test (FR), a versão do FR da posturografia, o Limite de Estabilidade (LE) e o TOS. Entre os grupos não houve diferença significante para o Functional Reach Test e o LE. No entanto, houve uma diferença significante entre os grupos para o TOS. O grupo que sofreu quedas teve uma menor média do escore da condição quatro.

Nos estudos de Buatois et al. ${ }^{22}$ e Ortuño-Cortés et al. ${ }^{23}$, o relato de quedas (número, circunstâncias e ferimentos) após a avaliação do equilíbrio corporal por meio de testes estáticos, dinâmicos e TOS da PDC e por testes funcionais (Timed Up and Go test, One-Leg Balance e Sit-to-Stand-test) foi acompanhado prospectivamente em 189 idosos (idade média de 70,5 anos) saudáveis e funcionalmente ativos, por um período de 16 meses (avaliações realizadas a cada quatro meses). Foi verificado, neste estudo, que 132 (70,0\%) idosos não relataram quedas no seguimento, $38(20,0 \%)$ relataram uma queda e 19 (10,0\%) duas ou mais quedas.

Não houve diferença significativa entre os três grupos (não caiu, caiu uma vez, caiu duas vezes ou mais) quando comparados quanto às variáveis: idade, gênero, autoavaliação do estado atual de saúde, atividade física, índice de massa corporal, avaliação cognitiva, medo de queda e a história prévia de queda. Os autores observaram diferença para o número de medicamentos utilizados ( $\mathrm{p}=0,057)$, sendo maior a utilização no grupo de caidores recorrentes. Os dados da pesquisa não identificaram diferença significante entre os grupos nos estáticos e dinâmicos da PDC e nos testes funcionais ${ }^{22}$

Entretanto, em relação aos resultados do TOS da PDC, o escore de equilíbrio da condição dois (superfície fixa e OF, e a análise somatossensorial) apresentaram diferença significante à comparação dos três grupos. Os caidores recorrentes apresentaram pior desempenho em relação aos idosos que caíram uma vez e, estes apresentaram melhor $\mathrm{CP}$ em relação aos que não caíram. As medidas, segundo os autores, que diferenciaram melhor o equilíbrio corporal, em conjunto por análise de regressão logística, entre idosos com quedas recorrentes e que não caíram, foram a terceira tentativa da condição seis (plataforma móvel e campo visual móvel) e a análise somatossensorial ${ }^{22}$.

Piirtola e $\mathrm{Era}^{14}$ realizaram uma revisão sistemática de estudos prospectivos mostrando que a velocidade média ML e a amplitude do deslocamento ML do COP na posição ortostática em SF com $\mathrm{OA}$ ou $\mathrm{OF}$ foram preditoras de quedas em idosos quando mensuradas em plataforma de força. As medidas destes parâmetros na posturografia dinâmica de acordo com os autores da pesquisa não foram consideradas preditivas de quedas em idosos ${ }^{14}$.

Em 2008, Ortuño-Cortés, Martín-Sanz e Barona-de Guzmán ${ }^{23}$, realizaram a avaliação da área $\left(\mathrm{mm}^{2}\right)$, velocidade média $(\mathrm{m} / \mathrm{s})$, dispersão $\mathrm{ML}$ e $\mathrm{AP}(\mathrm{mm})$ e deslocamento $\mathrm{ML}$ e AP máximos $(\mathrm{mm})$ na posturografia estática do $\mathrm{NedSVE} / \mathrm{IBV}^{\circledR}$, em quatro condições (Romberg com OA, com OF, com OA em espuma, e OF em espuma). Esta avaliação foi feita em 120 pacientes idosos entre 65 a 80 anos (60 idosos sem distúrbios vestibulares - grupo controle e 60 com distúrbios do equilíbrio, sendo 48 com distúrbios periféricos compensados e descompensados e 12 com distúrbios centrais). Os resultados mostraram um melhor desempenho do grupo controle em todos os testes. Os pacientes com distúrbios centrais tiveram resultados piores que os idosos com doença periférica e, destes, os pacientes descompensados foram piores. Os valores de oscilação aumentaram, respectivamente, nas condições de Romberg com OA, Romberg com OF, Romberg com os OA em espuma e Romberg com OF em espuma. Todos os parâmetros estudados (exceto velocidade média) foram capazes de discriminar o estado de compensação dos pacientes com doença vestibular periférica na condição de Romberg em espuma com OA. Nenhum dos parâmetros estudados na condição sobre espuma com OF discriminou pacientes de acordo com o estado de compensação vestibular ${ }^{23}$. 
Além disso, todos os resultados da posturografia estática (exceto para o parâmetro de velocidade média) correlacionaram significativamente com os resultados da Berg Balance Scale nas condições de Romberg com OA e com OF, e sobre a espuma com AO. Segundo os autores, estes resultados parecem ser mais adequados do que a condição de espuma com OF para explicar o desempenho funcional do equilíbrio na faixa etária de 65 a 80 anos. Correlações significantes foram identificadas entre os resultados da posturografia na condição de Romberg sobre a espuma com OA e o desempenho nos testes clínicos (Berg Balance Scale, apoio unipodal direito e esquerdo, subescalas estática e dinâmica de Tinetti e Time Up and Go Test). A partir destes achados pode ser indicado que, de todas as condições estudadas, esta é a que melhor reflete o estado de compensação vestibular e clínico-funcional da amostra estudada ${ }^{23}$.

Pedalini et al..$^{13}$ avaliaram o CP de 58 adultos (média etária de 33 anos), de 60 idosos (média etária de 69,3 anos) sem sintomas vestibulares, e também de 60 idosos (média etária de 70,1 anos) com tontura e/ou desequilíbrio corporal de origem vestibular. Para tanto, utilizaram o TOS da PDC, nas seis condições sensoriais. Os resultados posturográficos encontrados foram piores no grupo de idosos sintomáticos quando comparados ao grupo de idosos sem queixa, revelando que as alterações vestibulares associadas à senescência comprometem o CP; os resultados do grupo de idosos sem queixa foram piores em relação ao grupo de adultos saudáveis, mostrando o comprometimento dos sistemas visual e vestibular no processo de envelhecimento.

A realidade virtual é usada na Medicina desde 1994 com diversos objetivos. Dentre eles, para induzir a oscilação visual nos métodos de posturografia, ou seja, um sistema integrado à realidade virtual com uma plataforma de força para a avaliação do $\mathrm{CP}^{24,25}$. A realidade virtual na avaliação do $\mathrm{CP}$ permite avaliar o impacto da percepção sensorial sobre respostas posturais quando estes estímulos visuais são oferecidos como, por exemplo, em pacientes com dependência visual, como ocorre frequentemente nos idosos. Desta forma, torna-se mais fácil compreender os limites da capacidade humana, determinar uma variação precisa de informações sensoriais e respostas posturais ${ }^{26}$.

Para explicar os diferentes papéis desempenhados pelo fator visual no desencadeamento da instabilidade, pacientes idosos com distúrbio vestibular central e histórico de desequilíbrio e duas ou mais quedas no último ano ( $\mathrm{n}=18 ; 72-84$ anos) foram submetidos a estímulos visuais e avaliados por meio da plataforma AMTI Accusway; um grupo de 24 voluntários (41-72 anos) foi selecionado como controle. As três condições de estimulação usadas foram: (1) postura ortostática, OA, (2) postura ortostática, OA e campo visual dinâmico (estimulação foveal), e (3) postura ortostática, OA e campo visual dinâmico (estimulação optocinética horizontal). Verificou-se que o $\mathrm{CP}$ dos 18 pacientes com distúrbio vestibular central não foi homogêneo, e, portanto, os pacientes foram subdivididos em Grupo A $(n=13)$ e Grupo B $(n=5)^{26}$.
No grupo principal (Grupo A), os valores médios da VOC e da área do COP foram maiores após a estimulação visual em relação ao grupo controle, porém a diferença não foi significante, pois os estímulos visuais podem atuar como fatores de risco para alteração de equilíbrio e quedas. No Grupo B, a estimulação optocinética apresentou aumento significantemente maior dos valores da VOC e da área do COP ( $<<0,010)$ em relação ao controle. Em quatro pacientes do Grupo B, a eletronistagmografia apresentou assimetria do nistagmo optocinético, com preponderância direcional significante, sugerindo uma possível relação entre o distúrbio deste reflexo e a resolução ruim do conflito visual no $\mathrm{CP}^{26}$.

$\mathrm{Na}$ avaliação pelo estabilograma, todos os pacientes do Grupo A ajustaram o $\mathrm{CP}$, principalmente na direção $\mathrm{Y}$ (para trás e para frente). Entretanto, os pacientes do Grupo B apresentaram distribuição de frequências de oscilação, tanto nas direções dos eixos $\mathrm{X}$ e Y (para frente, para trás e para laterais) durante a estimulação visual. Os pacientes do Grupo B apresentaram dispersão significativamente maior $(\mathrm{p}<0,01)$ dos valores nas velocidades, principalmente, quando submetidos à estimulação optocinética ${ }^{26}$.

A partir destes resultados, foram propostos três marcadores para indicar a dependência visual e risco de queda: 1 . aumento na área do COP em valores significativamente maiores $(>3,40$ e DP de $0,69 \mathrm{~cm}^{2}$ ) com estimulação optocinética; 2 . aumento significativo na $\operatorname{VOC}(>3,89$ e DP de $1,15 \mathrm{~cm} / \mathrm{s})$, com histograma mostrando distribuição de valores entre 0 (zero) e $18 \mathrm{~cm} / \mathrm{s} ; 3$. frequências de oscilação, distribuídas em ambos os eixos (X e Y) e estendido para frequências mais altas (até $3 \mathrm{~Hz}$ ) durante a estimulação visual com valores maiores que cinco. Portanto, a alteração destes três marcadores sugere maior risco de queda e dependência visual em alguns pacientes com síndrome vestibular central ${ }^{26}$.

$\mathrm{O} C P$ à $\mathrm{BRU}^{\circledR}$ e o histórico de instabilidade e quedas no último ano de 57 idosos (67-82 anos) foram estudados; 46 voluntários saudáveis (29-75 anos) foram avaliados como controle. Os valores do LE em pacientes com instabilidade e quedas $\left(174,00 \mathrm{~cm}^{2}\right.$ e DP de 26,00) foram significantemente inferiores aos valores médios da amostra de indivíduos normais $\left(330,52 \mathrm{~cm}^{2}\right.$ e DP de 32,00), mostrando uma "área segura reduzida" desenvolvida pela trajetória do COP no grupo de idosos que sofreram quedas, apesar da amostra do grupo controle ser mais jovem. Os idosos com instabilidade e quedas mostraram valores superiores da área do COP e da VOC nas condições avaliadas de estimulação sensorial (a SF, OA e campo visual estático; b. SF e campo visual dinâmico - perseguição ocular lenta, estímulos sacádicos, estímulos optocinéticos nas direções horizontal e vertical e IVV), principalmente nesta última, provavelmente, porque os idosos com desequilíbrio têm dificuldade para estabilizar a imagem na retina, inferindo comprometimento do $\mathrm{RVO}^{24}$.

$\mathrm{O}$ CP de idosos vestibulopatas crônicos, com ou sem histórico de quedas, foi aferido à posturografia estática integrada à realidade virtual $\left(B R U^{\circledR}\right)$, em dez condições sensoriais. Foram incluídos 117 idosos distribuídos em: grupo controle (GC) - saudáveis, e grupo vestibulopatas crônicos (G1-sem quedas; e G2-com 
quedas). Foi verificado que idosos saudáveis apresentaram LE melhor do que idosos vestibulopatas crônicos com ou sem quedas; idosos saudáveis apresentaram área do COP e VOC similares à de idosos vestibulopatas crônicos sem quedas e melhores em relação àqueles que apresentaram quedas. Idosos vestibulopatas crônicos sem quedas apresentaram LE similar, área do COP melhor e VOC similar em relação àqueles que apresentaram quedas; o CP de idosos vestibulopatas crônicos com quedas é pior do que o de idosos saudáveis e de vestibulopatas crônicos sem quedas ${ }^{27}$.

O LE é a área onde a oscilação do paciente é mais segura; uma redução significativa desta área faz com que qualquer oscilação súbita do COP possa exceder o LE e aumentar o risco de queda ${ }^{24}$. O LE é a área em que um indivíduo pode mover seu centro de massa corporal e manter-se em equilíbrio sem a mudança da base de apoio. Em pacientes idosos com alteração do equilíbrio corporal, este cone de estabilidade é frequentemente diminuído ou suas representações neurais centrais são distorcidas, as quais afetam a seleção de estratégias de movimento para manter o equilíbrio ${ }^{28}$. Os idosos com vestibulopatia crônica e risco para quedas apresentam redução da amplitude do limite de estabilidade LE e aumento da oscilação postural ${ }^{27}$. Entre os artigos encontrados nesta revisão somente Wallmann ${ }^{21}$, relatou dados avaliados do LE em comparação com o Functional ReachTest. Entre os grupos de idosos os autores não relataram diferença significante entre o Functional ReachTest e o LE.

Em condição de superfície firme e olhos abertos, a utilização de pistas visuais e somatossensoriais para a manutenção do equilíbrio corporal pode compensar uma informação imprecisa do sistema vestibular ${ }^{13}$. Em situações estáticas, os sistemas mais relevantes estão atuando abaixo de seus limiares e os mecanismos de CP não podem ser plenamente explorados ${ }^{22}$. Já em condição de superfície firme e olhos fechados, Baloh et al. ${ }^{7}$, Baloh et al. ${ }^{20}$, Piirtola e Era ${ }^{14}$ apontaram pior CP de idosos com histórico de quedas em relação aos que não caíram, mostrando que o uso da informação visual tem grande importância para o equilíbrio corporal, principalmente nesta população, que apresenta alterações somatossensoriais decorrentes do envelhecimento ${ }^{29,30}$.

Em condição de espuma com OA, a informação somatossenorial está imprecisa, pois as alterações decorrentes do envelhecimento, como a diminuição na sensação táctil na planta dos pés, aumento do limiar para sensibilidade vibratória e diminuição da acurácia no senso de posição $\operatorname{articular}{ }^{29}$ por si só, trazem prejuízos funcionais, como também alterações das respostas posturais à avaliação posturográfica, como demonstrado nos estudos de Piirtola e Era ${ }^{14}$ e Ortuño-Cortés, Martín-Sanz e Barona-de Guzmán²3.

Em condição de espuma com OF, as informações do sistema somatossensorial estão imprecisas pelo uso da espuma e a informação visual ausente, o sistema vestibular age como principal fonte de informação sensorial para o CP. Esta condição é claramente uma das mais desafiadoras para o $\mathrm{CP}$ em idosos com vestibulopatias $^{2}$. A informação somatossensorial inacurada e a retirada da informação visual fazem com que os indivíduos dependam da função vestibular para o CP, desencadeando aumento da oscilação corporal e maior risco de queda em idosos com vestibulopatias. Ao contrário, na condição 1 ( $\mathrm{SF}$ e $\mathrm{AO}$ ), condição basal da avaliação posturográfica Whitney, Marchetti e Schade ${ }^{15}$ e Pedalini et al. ${ }^{13}$, em relação à área do COP e a VOC os autores não mostraram alterações importantes nesta condição. Estes achados demonstraram valores normais do COP e VOC na condição sem estímulo sensorial para a maioria dos pacientes com disfunção vestibular crônica, uma vez que utilizam pistas visuais e somatossensoriais para manutenção do equilíbrio corporal, compensando a informação inacurada do sistema vestibular.

Dentre as investigações aqui revisadas algumas, apresentaram dados que reforçam uma relação positiva da idade avançada (como comprometimento visual e vestibular) com o comprometimento do controle postural dos indivíduos, a medida que as análises de interação com a variável gênero, não apresentou significância estatística $^{8,15}$. Além disso, nas pesquisas, onde a amostra contava com grupos de população jovem, piores escores relacionados às medidas de mensuração do TOS e VOC eram identificados quanto maior fosse a faixa etária ${ }^{8,13,15,19}$. Análise realizada por Pedalini et al. ${ }^{13}$ mostrou piores escores nos testes de posturografia, mesmo quando a comparação foi realizada entre idosos (com equilíbrio normal para a idade) e indivíduos mais jovens.

Nos sistemas de posturografias integradas à realidade virtual, a estimulação visual por meio dos óculos de realidade virtual age sobre os movimentos oculares reflexos como o rastreio pendular, sacádico, perseguição ocular lenta, vergência e optocinético ${ }^{22,29}$. Estes movimentos participam ativamente nos mecanismos de $\mathrm{CP}$, integrados em harmonia com as múltiplas e complexas conexões vestibulares, como as vestíbulo-cerebelares, vestíbulo-espinhais, vestíbulo-oculares e vestíbulo-corticais, e permitem a estabilização da imagem da cena visual externa na retina nas diversas situações de movimento em que o ser humano é submetido ${ }^{22}$.

O sistema sacádico consiste em movimentos rápidos dos olhos, quando o RVO e a perseguição ocular lenta não conseguem manter o objeto de interesse na fóvea, para visualizá-lo nitidamente ${ }^{31}$. No envelhecimento, há diminuição do ganho dos movimentos sacádicos ${ }^{32}$.

A resposta optocinética é uma combinação dos mecanismos de perseguição ocular lenta e sacádico; pode ser produzida por movimentos repetidos com o indivíduo estacionário e campo visual em movimento, indivíduo em movimento e campo visual estacionário, ou ambos ${ }^{33}$.

O RVO é de grande importância para a manutenção do equilíbrio corporal, por ser o único reflexo com velocidade compatível aos movimentos cefálicos de alta velocidade angular ${ }^{1}$. Trata-se de um reflexo que estabiliza as imagens na retina durante o movimento cefálico, ao produzir um movimento ocular na direção oposta, preservando a nitidez visual durante a locomoção ${ }^{32}$. Apresenta comprometimento relacionado à senescência vestibular ${ }^{1,31}$, decorrente da redução da sensibilidade dos canais semicirculares às estimulações angulares de alta 
frequência, interferindo nas respostas reflexas aos movimentos rápidos do segmento cefálico ${ }^{34}$.

Com o envelhecimento os sistemas sensoriais e motores relacionados com o equilíbrio corporal e suas adaptações, sofrem acúmulo de processos degenerativos, infecciosos e/ou traumáticos que comprometem seu funcionamento adequado ${ }^{35,36}$. De forma particular no sistema visual ocorre diminuição no número dos receptores proprioceptivos nos músculos oculares. Além disso, o sistema musculoesquelético que é responsável pelas ações musculares e desempenha papel fundamental no controle postural, pode apresentar perda de fibras musculares, neurônios motores, unidades motoras, força e massa muscular, o que provoca importante diminuição na capacidade de gerar força ${ }^{37,38}$. Embora a alteração isolada de um dos sistemas possa não apresentar impacto preponderante do desenvolvimento da instabilidade corporal, a somatória dessas deficiências é um fator determinante para o desequilíbrio corporal da população idosa ${ }^{39,40}$.

Os resultados do teste posturográfico devem ser avaliados em conjunto com a história clínica, o exame físico e testes laboratoriais ${ }^{9,26}$. A posturografia permite avaliar pacientes com distúrbios do equilíbrio corporal, tontura ou instabilidade e complementa os testes convencionais da avaliação otoneurológica, fornecendo informações não somente do sistema vestibular, mas dos demais sistemas que contribuem para a manutenção do equilíbrio corporal ${ }^{25}$.

A avaliação posturográfica computadorizada estática ou dinâmica permite identificar quais das condições sensoriais mais precisam ser enfatizadas na reabilitação do equilíbrio corporal de idosos com distúrbios vestibulares, pois apresentam maiores alterações dos parâmetros do CP (LE, VOC, área do COP), quando comparados aos idosos que não apresentam desequilíbrio corporal, tontura e/ou quedas e também aos indivíduos mais jovens. A reabilitação visa à integração sensorial e o aperfeiçoamento das estratégias do CP, buscando dessa forma, prevenir e/ou diminuir futuras quedas ou minimizar as consequências deletérias delas advindas, ressaltando que afetam consideravelmente os custos dos sistemas de saúde.

\section{REFERÊNCIAS}

1. Konrad HR, Girardi M, Helfert R. Balance and Aging. Laryngoscope. 1999;109(9):1454-60.

2. Suarez $\mathrm{H}$, Arocena M. Las alteraciones del equilibrio en el adulto mayor. Rev Med Clín Condes. 2009;20(4):401-7.

3. Horak FB. Clinical assessment of balance disorders. Gait Posture. 1997;6(1):76-84

4. Hageman PA, Leibowitz JM, Blanke D. Age and gender effects on postural control measures. Arch Phys Med Rehabil. 1995;76(10):961-5

5. Keshner EA. Anormalidades posturais nas disfunções vestibulares. In: Herdman SJ. Reabilitação Vestibular. 2a ed. São Paulo: Manole; 2002. p.52-71.

6. Mochizuki L, Amadio AC. Aspectos biomecânicos da postura ereta: a relação entre centro de massa e o centro de pressão. Rev Port Ciênc Desporto. 2003;3(3):77-83.

7. Baloh RW, Spain S, Socotch TM, Jacobson KM, Bell T. Posturography and balance problems in older people. J Am Geriatr Soc. 1995;43(6):638-44

8. Baloh RW, Jacobson KM, Enrietto JA, Corona S, Honrubia $V$. Balance disorders in older persons: quantification with posturography. Otolaryngol Head Neck Surg. 1998;119(1):89-92.

9. Bittar RSM. Como a posturografia dinâmica computadorizada pode nos ajudar nos casos de tontura? Arq Int Otorrinolaringol. 2007;11(3):330-3.

10. Cusin FS, Ganança MM, Gananç FF, Ganança CF, Caovilla HH. Balance Rehabilitation Unit (BRU) posturography in Menière's disease. Braz J Otorhinolaryngol. 2010;76(5):611-7.

11. Di Fabio RP. Sensitivity and specificity of platform posturography for identifying patients with vestibular dysfunction. Phys Ther. 1995;75(4):290-305
12. Di Fabio RP. Meta-analysis of the sensitivity and specificity of platform posturography. Arch Otolaryngol Head Neck Surg. 1996;122(2):150-6.

13. Pedalini ME, Cruz OL, Bittar RS, Lorenzi MC, Grasel SS. Sensory organization test in elderly patients with and without vestibular dysfunction. Acta Otolaryngol. 2009;129(9):962-5.

14. Piirtola M, Era P. Force platform measurements as predictors of falls among older people: a review. Gerontology. 2006;52(1):1-16.

15. Whitney SL, Marchetti GF, Schade Al. The relationship between falls history and computerized dynamic posturography in persons with balance and vestibular disorders. Arch Phys Med Rehabil. 2006;87(3):402-7.

16. Blaszczyk JW, Orawiec R, Duda-Klodowska D, Opala G. Assessment of postural instability in patients with Parkinson's disease. Exp Brain Res. 2007;183(1):107-14.

17. Shivitz N, Koop MM, Fahimi J, Heit G, Bronte-Stewart HM. Bilateral subthalamic nucleus deep brain stimulation improves certain aspects of postural control in Parkinson's disease, whereas medication does not. Mov Disord. 2006;21(8):1088-97.

18. Cohen H, Heaton LG, Congdon SL, Jenkins HA. Changes in sensory organization test scores with age. Age Ageing 1996;25(1):39-44

19. Furman JM. Role of posturography in the management of vestibular patients. Otolaryngol Head Neck Surg. 1995;112(1):8-15.

20. Baloh RW, Corona S, Jacobson KM, Enrietto JA, Bell T. A prospective study of posturography in normal older people. J Am Geriatr Soc. 1998;46(4):438-43.

21. Wallmann HW. Comparison of elderly nonfallers and fallers on performance measures of functional reach, sensory organization, and limits of stability. J Gerontol A Biol Sci Med Sci. 2001;56(9):M580-3 
22. Buatois S, Gueguen R, Gauchard GC, Benetos A, Perrin PP. Posturography and Risk of Recurrent Falls in Healthy Non-Institutionalized Persons Aged Over 65. Gerontology. 2006;52(6):345-352

23. Ortuño-Cortés MA, Martín-Sanz E, Barona-de Guzmán R. Static posturography versus clinical tests in elderly people with vestibular pathology. Acta Otorrinolaringol Esp. 2008;59(7):334-40.

24. Suarez H, Arocena M, Geisinger D, Suarez A, Carrera X. Analysis of the role of virtual reality technology in the assessment and rehabilitation of Instability in the elderly population. In: Vincent ML, Moreau TM. Accidental falls: causes, preventions and interventions. Nova York: Nova Science; 2008. p. 107-20.

25. Gazzola JM, Doná F, Ganança MM, Suarez H, Ganança FF, Caovilla HH. Realidade virtual na avaliação e reabilitação dos distúrbios vestibulares. Acta ORL. 2009;27(1):22-7.

26. Suarez H, Musé P, Suarez A, Arocena M. Assessment of the risk of fall, related to visual stimulation, in patients with central vestibular disorders. Acta Otolaryngol. 2001;121(2):220-4.

27. Gazzola JM. Controle postural de idosos vestibulopatas crônicos com e sem histórico de quedas submetidos à estimulação visual por realidade virtual. Tese [Doutorado]. São Paulo: Universidade Federal de São Paulo; 2010.

28. Matsumura BA, Ambrose AF. Balance in the Elderly. Clin Geriatr Med. 2006;22(2):395-412

29. Booth FW, Weeden SH, Tseng BS. Effect of aging on human skeletal muscle. And motor function. Med. Sci. Sports exerc. 1994;26(5):556-60.

30. Thornell LE, Lindstrom M, Renault V, Mouly V, Butler Browm GS. Satellite cell and training in the elderly. Scand J Med Sci Sports. 2003;13(1):48-55
31. Lewis RF, Zee DS, Goldstein HP, Guthrie BL. Proprioceptive and retinal afference modify postsaccadic ocular drift. J Neurophysiol. 1999;82(2):551-63

32. Konrad HR, Girardi M, Helfert R. Balance and Aging Laryngoscope. 1999;109:1454-60

33. Shepard NT. Evaluation of the patient with dizziness and balance disorders. In: Katz J, Medwetsky L, Burkhart B. Handbook of clinical audiology. 6th ed. Media PA: Williams \& Wilkins; 2007.

34. Nakayama M, Helfert $\mathrm{RH}$, Konrad HR, Caspary DM. Scanning electron microscopic evaluation of age-related changes in the rat vestibular epithelium. Otolaryngol Head Neck Surg. 1994;111(6):799-806.

35. Horak FB, Hlavacka F. Somatossensory loss increases vestibulospinal sensitivity. J Neurophysiol. 2001;86(2):575-85.

36. Gazzola JM, Ganança FF, Aratani MC, Perracini MR, Ganança MM. Clinical evaluation of elderly people with chronic vestibular disorder. Braz J Otorhinolaryngol. 2006;72(4):515-22

37. Mitchell PD, Mitchell TN. The age-dependent deterioration in light touch sensation on the plantar aspect of the foot in a rura community in India: implications when screening for sensory impairment. Lepr Rev. 2000;71(2):169-78.

38. Richardson JK, Ashton-Miller JA. Peripheral neuropathy: an often-overlooked cause of falls in the elderly. Postgrad Med. 1996;99(6):161-72

39. Norré ME. Posture in otoneurology. Acta Otorhinolaryngol Belg. 1990;44(2):55-181

40. Gassmann KG, Rupprecht R. Dizziness in an older community dwelling population: a multifactorial syndrome. J Nutr Health Aging. 2009;13(3):278-82. 